\title{
3 Research Square \\ Bedside Diffuse Optical Tomography of Disrupted Brain Connectivity During Acute Stroke
}

Broc Burke ( $\square$ broc@stanfordalumni.org )

Washington University School of Medicine https://orcid.org/0000-0003-2825-3627

Karla Bergonzi

L3Harris

Arefeh Sherafati

Washington University

Andrew Fishell

Washington University School of Medicine

Tracy Burns-Yocum

Indiana University

Silvina Ferradal

Indiana University

Ben Palanca

Washington University School of Medicine

Rajat Dhar

Washington University School of Medicine

Gyanendra Kumar

Mayo Clinic

Jin-Moo Lee

Washington University in St. Louis

Joseph Culver

Washington University

Adam Eggebrecht

Washington University https://orcid.org/0000-0002-6320-2676

Article

Keywords: Functional Connectivity, Infrared, Neuroimaging, NIH Stroke Scale, Non-invasive

Posted Date: December 2nd, 2020

DOI: https://doi.org/10.21203/rs.3.rs-107369/v1 
License: (c) (i) This work is licensed under a Creative Commons Attribution 4.0 International License. Read Full License 


\section{Abstract}

Neurological instability during the first days following ischemic stroke reflects the potential to salvage brain tissue at risk for infarct growth, hemorrhagic transformation, or malignant edema. However, current clinical care provides intermittent assessments on the order of at least an hour apart potentially missing the early detection of neurological deterioration. Optical imaging offers a continuous, portable alternative to the current combination of physical exams and radiological imaging. However, optical imaging technology has heretofore lacked the crucial combination of portability, resolution, and coverage required for assessing distributed brain function. Here, we present a portable high-density diffuse optical tomography (HD-DOT) system that overcomes these limitations to assess neurological disruption at the bedside. We imaged patients within 72 hours after ischemic stroke onset and demonstrate that HD-DOT is sensitive to both the presence of stoke (Cohen's $d=1.5, p=1.2 \times 10^{-3}$ ) as well as the degree of neurological disruption measured by NIH Stroke Scale $\left(R^{2}=0.69, p=4.7 \times 10^{-4}\right)$.

\section{Significance Statement}

Ischemic stroke, caused by insufficient oxygen delivery to the brain, ignites a variable series of events that acutely unfold over the first three days and that can lead to permanent dysfunction and neurological impairment. Current bedside monitoring methods are limited to intermittent physical exams that may miss deteriorating physiology. Here, we present integrative advancements in portable high-density diffuse optical tomography (HD-DOT) that enabled bedside assessments of brain health during the first 72 hours of stroke recovery. Our results demonstrate efficacy for HD-DOT as a bedside tool that is sensitive to the severity of functional disruption in the brain. Quantitative bedside monitoring of brain function in patients with neurologic deficits over extended periods of time may revolutionize clinical care during recovery.

\section{Introduction}

One in 35 people in the United States experience a stroke in their lifetime. This number is expected to increase to nearly 1 in 25 by the year 2030, making stroke the leading preventable cause of disability ${ }^{1}$. Ischemic stroke, the major stroke subtype, typically begins with the occlusion of an artery in the brain 2, 3 . This occlusion triggers a complex and cascading series of events including anoxic depolarization, excitotoxicity, spreading depression, and, in some cases, reperfusion. Brain injury evolves rapidly during the first 24 hours after onset, and therapeutic interventions aim to preserve viable brain tissue 4, 5,6. Beyond this acute phase, potential complications include clot propagation with infarct growth, hemorrhagic transformation, and life-threatening cerebral edema ${ }^{7}$. Therefore, during the first days after stroke onset, early detection of neurological deterioration is essential and close neurological monitoring is critical. For critically ill stroke patients, current standard of care consists of serial vital sign monitoring and neurological exams at approximately 2 -hour intervals ${ }^{2}$. If neurological deteriorations occur, it is often not detected until the designated neuro exam is performed. An imaging modality that continuously measures brain function at the bedside could potentially provide near-real-time monitoring to better 
assess changes in neurological status and inform clinical decisions earlier. Due to current technological and logistical limitations, CT and MRI scanners cannot fulfill this role.

Optical imaging has long held promise as a bedside neuroimaging technique due to small console size and relative ease of use ${ }^{8}$. However, reliability and image quality of bedside-deployable optical imaging systems has been lacking, particularly in comparison to functional magnetic resonance imaging (fMRI) ${ }^{9}$, 10,11, 12, 13, 14 . Recent developments in High-Density Diffuse Optical Tomography (HD-DOT ${ }^{15}$ ) have demonstrated dramatically improved image quality relative to sparse optical systems and have been shown to produce fMRI-comparable maps of brain function ${ }^{16,17,18}$. Importantly, extant research has also established the efficacy of HD-DOT to map functional connectivity $(\mathrm{fc})$ of spatially distributed resting state networks in the absence of tasks in adults $9,19,20,21,22,23$ and neonates ${ }^{18,24}$. Obtaining high-fidelity fc maps requires high-sensitivity and low-noise opto-electronics along with a spatially distributed imaging array that have heretofore been limited to laboratory settings in studies in adult participants. Indeed, expanding the FOV of HD arrays to cover a significant portion of the head while maintaining a portable HD-DOT console presents significant challenges in imaging array ergonomics, optical-fiber-scalp coupling, and real-time data quality assessment.

In this study, we present a portable HD-DOT imaging system that overcomes these technical challenges through integrative advances in fiber optic imaging cap design, novel algorithms for real-time evaluation of data quality at the bedside, and analysis strategies to compare the rich functional connectivity DOT (fcDOT) data with clinically relevant behavioral metrics of stroke-induced dysfunction. To minimize heterogeneity in the clinical study population, this study focused on patients with an occlusion of the middle cerebral artery (MCA) territory. Spatio-temporal intrinsic brain activity was collected as the patients rested quietly in their inpatient bed. Bilateral cortical imaging maps of fcDOT across thousands of regions of interest were evaluated against those collected from a healthy control population. We demonstrate that the performance of the reported HD-DOT system coupled with fcDOT analysis strategies enables mapping of disruption in spatially distributed fc patterns at the clinical bedside within the first 72-hours following an ischemic occlusion. Further, we show that the degree of disruption in brain function correlates significantly with quantitative neurobehavioral clinical stroke measures acquired at the time of imaging. Collectively, our study demonstrates that advanced fcDOT imaging methods offer a practical and robust complement to CT or MRI assessment of functional brain health. Furthermore, bedside monitoring of neurologically impaired and even unresponsive adult patients over extended periods of time may revolutionize clinical care during the acute phase of recovery from ischemic stroke.

\section{Results}

The clinical HD-DOT console and imaging cap facilitated bedside imaging within the intensive care unit of adults during the initial 72-hour acute phase of recovery from ischemic stroke (Fig. 1). The imaging cap was constructed to enable strong and efficient optical coupling with the scalp through the hair over bilateral aspects of cortex expected to exhibit functional disruptions given an ischemic insult to the MCA 
watershed (Fig. 1). To ensure optimal fit of the imaging array on the patients, multiple metrics of real-time data quality were presented to the imaging team at the bedside. Key metrics for real-time data fidelity included coupling coefficients for each optical fiber with the scalp (Fig. 2a), the temporal variance (cut-off $>7.5 \%$ ) (Fig. 2b), and fall-off of mean light intensity of each measurement against source-detector distance (Fig. 2C), and cardiac pulse signal to noise across the imaging array (Fig. 2d). Together, these metrics reflect the log-linear light fall-off characteristic inherent to photon diffusion through biological tissue and a strong pulse signature indicative of optical data with a high signal to noise ratio at the 82 points of optical contact that support up to 880 optical measurements.

Brain functional connectivity was assessed throughout the optically-accessible field of view using a seedbased region of interest (ROI) strategy (Fig. 3). Disruption in multiple functional connectivity maps (fcmaps) is apparent at the individual subject level, as shown in the fc-maps of three stroke patients generated by ROI placement over right motor or right visual cortex. Specifically, in the older control participant, fCDOT maps demonstrated strong interhemispheric correlation between homotopic counterparts for each of the right motor and right visual ROIs, indicating healthy and normal functional connectivity. By contrast, in the stroke patients, the correlation values contralateral to the right motor ROI diminish in a manner that correlates with the stroke severity as assessed via either the volume of infarcted tissue or the magnitude of the National Institutes of Health Stroke Scale (NIHSS).

To quantify disruption in $\mathrm{fc}$ throughout the FOV, we calculated the spatial correlation between a given ROI-based fc-map of a stroke patient and an equivalent ROI-based mean fc-map of a young healthy reference population (Fig. 4; see Methods). This spatial correlation is termed the similarity. Due to the strongly heterogeneous nature of MCA ischemic stroke location and size, we took a data-driven approach and assessed the similarity of the fc-maps utilizing an ROI centered at each voxel containing cortical tissue, generating $n=10,241 \mathrm{fc}$-maps per patient. To help control for the heterogeneity in the patient population, datasets with infarcts in the right hemisphere were flipped across the sagittal axis in order to have all infarcts located in the left hemisphere. To account for age and then assess the effect of stroke on the similarity of the fc-maps, we also calculated the similarity for each of the older controls relative to the young adult reference group. As each cortical voxel has an associated similarity value for each participant, a map of the mean similarity was generated across participants in each group (Fig. 5a). The similarity values for stroke patients are significantly lower than values for older healthy controls (stroke $n$ $=13$, healthy $n=11$, independent samples $t$-test, $\left(t=3.7, p<1.2 \times 10^{-3}\right)$, and the group difference in mean similarity value exhibits a strong effect size (Cohen's $d=1.5$, Fig. $5 a$ and $5 b$ ).

Observation of the within-patient distribution of similarity values reveals a remarkable increase in skewness magnitude towards stronger negative values as the stroke severity increases (Fig. $5 \mathrm{c}$ and $5 \mathrm{~d}$ ). To assess the relationship between a behavioral measure of stroke severity and fcDOT, we calculated the Pearson correlation between the NIHSS and the skewness of the similarity distribution of each stroke patient. We found that the skewness of the similarity values is strongly negatively correlated with the NIHSS $\left(n=13, r=-0.83, R^{2}=0.69, p<4.7 \times 10^{-4}\right.$, Fig. $\left.5 d\right)$. In contrast to the skew, we found low and insignificant correlation of the mean value of the distribution with NIHSS $\left(n=13, r=0.15, R^{2}=0.02, p<\right.$ 
0.62). Finally, a significant positive relationship between the CT infarct volume and the NIHSS was quantified by Pearson correlation $\left(n=9, r=0.713, R^{2}=0.509, p=0.031\right)$, while the Pearson correlation between the similarity skewness and the infarct volume did not suggest collinearity $\left(n=9, R^{2}=0.030, p=\right.$ $0.653)$.

\section{Discussion}

Our results show disruptions in functional connectivity maps measured at the bedside in acute stroke patients when compared to older controls. Further, these results show a strong correlation between disruption in fc and a standard behavioral metric of neurological deficit, the NIHSS. During the first 72 hours after stroke onset, a patient's neurological status can be highly unstable. The logistics of traditional brain imaging such as $\mathrm{FMRI}$ and $\mathrm{CT}$ have hindered continuous investigation during this critical stage. We show that HD-DOT is well-suited for imaging during this time period because the system is portable and wearable while still covering an informative amount of the cortical surface for patients with ischemic events within the MCA watershed (Fig. 1c, d, and Fig. 3). Patient comfort, as optimized by the right-angle design of the fiber tips and the management of the fiber weight, were of utmost importance to obtaining high quality data (Figs. 1a, b). In addition, the real-time monitoring of HD-DOT data fidelity helped preserve multiple datasets by assisting the user in ensuring strong and stable fiber-scalp coupling (Fig. 2).

Intrinsic brain activity, often mapped using resting-state functional connectivity, provides an exquisitely sensitive indicator of the brain's physiological status. Altered functional connectivity has been documented in a wide variety of neurological and psychiatric conditions ${ }^{21,25}$. Studies assessing changes in functional connectivity as measured with MRI (fcMRI) have shown brain connectivity disruption in human stroke in the post-acute ( $>2$ weeks) phase $11,12,26,27$. However, fcMRI requires transporting patients to the MRI scanner. Therefore, serial fcMRI cannot feasibly be used to detect deteriorating neurological status in ICU patients. A non-invasive bedside method, such as HD-DOT, would enable serial evaluation of functional networks of stroke patients. We show that HD-DOT has the ability to map functional connectivity in acute stroke patients with proper data quality management such as real-time data assessments of optode-scalp coupling, physiology-based signal-to-noise, and motion detection (Fig. 2). Less severe injuries, as measured by a low NIHSS, showed intact functional connectivity maps that qualitatively look similar to the healthy control subject maps (Fig. 3, top two rows). Functional connectivity maps from patients with a higher NIHSS are qualitatively disrupted; however, due to variability in infarct locations, a trend in disruption is challenging to quantify. This finding is similar to those in the literature of decreased interhemispheric $\mathrm{fc}$ of the motor seed with increased damage to the corticospinal tract ${ }^{11}$. Other studies have shown correlates of $\mathrm{fc}$ with neurological deficit but only with specific seeds and infarct locations ${ }^{28}$. This variation highlights the challenges associated with characterizing a globally applicable neural signal associated with stroke-induced behavioral outcome. 
Using multiple fc-maps spanning numerous areas of the brain, we have developed a metric that is independent of infarct location. This metric, called the similarity metric, measures how similar a given fcmap of a stroke patient is to the map of a healthy subject. By calculating the similarity metric for multiple fc-maps across the brain, we sample both healthy and infarcted tissue. We found that stroke patients have lower average similarity values than the older healthy volunteers control cohort (independent samples t-test $=3.7$, Cohen's $d=1.5, p<1.2 \times 10^{-3}$, Fig. $5 b$ ). In evaluating correlation to NIHSS, we did not see a significant correlation between the mean value of the similarity over the FOV to NIHSS $(n=13, r=$ $\left.0.15, R^{2}=0.02, p<0.62\right)$. However, we did find that the skew of the similarity values is significantly correlated with the NIHSS $\left(p<4.7 \times 10^{-4}\right.$, Fig. $\left.5 d\right)$. The negative skew indicates that the stroke patients' fcmaps contain relatively more correlation values below the median, possibly due to hemodynamic delays in the brain's vascular supply ${ }^{29}$. This finding could potentially serve as a surrogate for neurological exams, allowing for continuous monitoring of cerebral function.

Implementing continuous monitoring over the first 72 hours and investigating how closely the similarity metric correlates with the NIHSS is an important next step in validating this metric. Further, investigating the information captured by the HD-DOT system could produce additional metrics to measure neuronal health. For example, this study focused only on the oxygenated hemoglobin signal. Future studies could investigate the deoxygenated and total hemoglobin signals, and how they each relate to each other. Additionally, while the similarity metric has the benefit of being independent of the infarct location, we have the possibility of studying the hemodynamic fluctuations specifically within the damaged tissue. Comparing anatomical and functional aspects of the infarct could provide helpful information for a clinician's treatment plan. Furthermore, the real-time monitoring potential of this technology can aid in the timeliness of clinical decision making both in traditional ICU settings as well as the remote ICU monitoring, which is becoming more commonplace.

In conclusion, clinical bedside application of fcDOT enables accurate assessment of network-specific patterns of functional connectivity dysfunction in patients during the acute stage of recovery from stroke. As a group, the functional connectivity maps of the patient group are significantly less similar to a healthy reference group than that of an older control group. This lower similarity reflects disruption in ultra-slow correlations of brain activity throughout the optically accessible field of view. Further, the magnitude of the skewness of the distribution of similarity values within a patient is correlated with the degree of neurological dysfunction as assessed with the NIHSS, a standard behavioral instrument. These advances in acquisition and analysis methods enable successful and relatively faster collection of high quality fCDOT data in patients in any medical setting, from the neurological ward to the ICU. Establishing the relationship between $\mathrm{fCDOT}$ results, structural neuroimaging findings, and cognitive and behavioral outcome measures may provide novel optical imaging biomarkers of brain injury and adverse outcomes that enable significant improvements in clinical care.

\section{Materials And Methods}




\section{Study Design and Participants}

The research was approved by the Human Research Protection Office at Washington University School of Medicine. This study was a prospective observational cohort study using a convenience sample. Three different groups were recruited for the study: 1) stroke patients, 2) older healthy, neurologically normal subjects to be used as controls, and 3) young healthy subjects to be used as a reference population.

We enrolled ischemic stroke patients who met the following enrollment criteria. Inclusion criteria included: 1) age greater than 18 years, 2) anterior circulation ischemic stroke with onset within 72 hours, and 3) $\mathrm{NIH}$ Stroke Scale (NIHSS) greater than or equal to 1 at time of initial evaluation in ER. Exclusion criteria included: 1) symptoms suggestive of a small sub-cortical stroke, 2) bilateral strokes, 3) prior stroke, 4) pregnancy, and 5) enrollment in an experimental therapeutic trial. Patients who met the inclusion and exclusion criteria were approached for consent. If the patient had difficulty with language production or comprehension due to the stroke, consent was obtained from the patient's legally authorized representative. Of those enrolled, 25 were excluded from analyses: one due to hair incompatibility, seven due to discomfort, five due to excessive movement, seven due to physician or family visits, two due to poor data quality caused by snoring, and three with less than 15 minutes of acquired data. We used a cutoff of 15 minutes of clean resting state data to optimize fidelity of the fc-maps at the level of the individual participant ${ }^{30,31}$. Of the remaining patients $(n=13$ with four females, mean age $=73.4( \pm 13.5)$ years), four had right-sided MCA strokes.

We recruited two control groups with a sole exclusion criterion of diagnosed neurological or psychological conditions. Nine young healthy volunteers were enrolled through an inclusion criteria of age between 18 and 30 years. Of these nine subjects, seven are used in this study to generate an average young healthy reference baseline with 2 being excluded due to motion artifacts limiting the total usable data to less than 15 minutes $(n=7$, mean age $=25.9( \pm 1.7)$ years. Similarly, 11 older normal control subjects were recruited with the inclusion of age greater than 55 years $(n=11$, mean age $=61.1( \pm 5.6)$ years).

To measure stroke severity, a National Institute of Health Stroke Scale (NIHSS) score evaluation was performed at the time of the HD-DOT scan. The NIHSS is a quantitative bedside assessment of neurological deficits, scoring disruptions in several different domains, including consciousness, vision, language, neglect, motor (extraocular, upper extremity, and lower extremity) and sensory ${ }^{32}$. These domains have been correlated with fMRI fc within the default mode network, the visual network, the fronto-parietal network, the dorsal attention network, and the somatomotor network respectively 10, 13, 14, 33, 34. Some patients underwent a structural head CT or brain MRI as part of their standard clinical care (see below). The bedside HD-DOT assessment did not interrupt standard clinical care.

\section{Clinical HD-DOT System Console}

The clinical HD-DOT console was designed to support high-fidelity measurement of light levels at the bedside in a small form-factor that would present no potential disruption to standard clinical care in the 
intensive care unit. The entire console of the system fit on a custom-built hospital-compliant console (Minitec, New York) measuring 4 feet in height, 3 feet in length, and 2 feet in width. This small footprint allowed for transport through hospital rooms for data collection without disrupting clinical care. The clinical HD-DOT console supported beside measurements arising from 48 source and 34 detector positions placed bilaterally in two rectangular grids on the participant's head (Fig. 1). The continuous wave HD-DOT system contained LED sources, illuminating with near-infrared (NIR) wavelengths $750 \mathrm{~nm}$ and $850 \mathrm{~nm}$ (750-03AU and OPE5T 85, Roithner Lasertechnik), and avalanche photodiode (APD) detectors (Hamamatsu C5460-01). Source boxes $(n=6)$ regulated power and delivered source encoding flashing patterns to 32 LEDs each (three $750 \mathrm{~nm}$ and one $850 \mathrm{~nm}$ LED per source position). The NIR light from the LEDs was directly coupled into 4 - 1 optical fiber bundles (CeramOptec, silicone cladded, $2.5 \mathrm{~mm}$ diameter bundles of $50 \mu \mathrm{m}$ fiber, numerical aperture 0.66 ) via SMA connectors. Light collected from the head was transmitted to detector boxes ( $n=6$, each housing up to six APD detectors) using similar 1-1 optical fibers and SMA connectors. The APDs were digitized by dedicated ${ }^{15} 24$-bit analog-to-digital converters at $96 \mathrm{kHz}$ (M32AD, RME). The system utilized temporal, frequency, and spatial encoding to sample the entire field of view at a $10 \mathrm{~Hz}$ framerate ${ }^{35}$. Specifications of the light level of the sources and noise levels of the detection opto-electronics have been previously described ${ }^{35}$.

\section{Clinical HD-DOT Imaging Cap}

The HD-DOT imaging cap was designed with two central goals: (i) to provide stability for efficient and reliable optical coupling, and (ii) to maximize ergonomic comfort for prolonged scanning sessions at the bedside in hospital patients. The custom optical fibers were manufactured with right-angle tips to couple into a soft and flexible neoprene-based imaging cap (Fig. 1b) that allowed for the patient to recline comfortably in their bed during an imaging session. The neoprene provided both a locally rigid structure, which maintained the regular HD grid, and flexibility that promoted fiber-scalp conformity for a wide variety of head shapes and sizes. Each of the 82 optical fiber tips was guided by a 'top-hat' style spacer to provide modest translation perpendicular to the head surface to facilitate consistent direct fiber-scalp coupling. Elastic rubber strips on the outside of the cap, held in place with plastic rivets, maintained pressure on the right-angle tips to ensure adequate coupling of the optical fiber tip with the scalp (Fig. 1b) while also maintaining a $\sim 3 \mathrm{~mm}$ penetration length of the optical fiber tip within the cap to ensure the tips comb through hair. The cap was attached to the patient using comfortable hook-and-loop straps across the forehead and over the top of the head. The imaging cap supported an HD imaging array by maintaining the 48 sources and 34 detectors in two interlaced rectangular arrays with first- through fourth-nearest neighbor separations as follows: 1.3, 3.0, 3.9, and $4.7 \mathrm{~cm}$ (Fig. 1c), with as many as 124, 170,54 , and 92 usable source-detector measurements per wavelength at the respective distances (up to 440 total measurements per wavelength). This arrangement produces a spatial resolution of approximately $13 \mathrm{~mm}$ within $1.0 \mathrm{~cm}$ below the cortical surface (Fig. 1c) ${ }^{36}$. To maximize lateral coverage over the MCA watershed area, the grid was designed with left and right panels (each containing 24 sources and 17 detectors) that were symmetrically oriented to each other relative to midline. The field of view of this HD-DOT array encompassed bilateral aspects of temporal, occipital, parietal, and prefrontal cortices (Fig. 1c,d). This coverage resulted in sensitivity to brain function within multiple key functional 
networks and much of the infarcted cortical tissue of the patients within this study. An infarct incidence map of the 10 patients with identifiable infarcts on either clinical MRI or clinical CT imaging shows damaged cortical tissue co-localized within the HD-DOT clinical field of view (Fig. 1d).

\section{Fitting the HD-DOT Imaging Cap}

To reliably acquire adequate coupling across the entire imaging cap, a simple set of steps were followed. First, the center of the cap was placed against the back of the head with the bottom row of fibers on the inion and angled such that the sides of the cap were situated approximately $0.5 \mathrm{~cm}$ above the helix-scalp intersect. Second, the cap was gently rubbed back and forth to comb the optical fiber tips through the patient's hair and to obtain stable coupling against the scalp for all 82 fiber tips. Third, two hook and loop straps were secured over the eyebrows of the patient to secure the cap to the head. Next, the fibers from each side of the imaging cap were gently guided over the head to help the cap match the curvature of the head. The weight of the fibers was supported by the bed around the patient and was neither disruptive nor uncomfortable. The position of the left and right pieces of the cap were checked for symmetric placement with the third source from the front placed just above the tragus at the dorsal-anterior earscalp connection.

\section{Real-time HD-DOT Data Quality Assessment}

Real-time metrics of data quality were presented on a computer monitor on the HD-DOT console to facilitate efficient at-the-bedside optimization of the cap coupling and position (Fig. 2). First, a schematic of the spatial layout of the optical coupling coefficients for each source and detector position helped localize specific optical elements that were not optimally coupled (Fig. 2a). These fibers could be directly accessed by the user to improve combing through the hair and strengthen the coupling at the scalp interface. Next, source-detector measurement pairs passing a temporal noise-to-signal ratio (NSR) threshold of $7.5 \%$ or less were displayed as green lines on a similar spatial layout as the coupling coefficients (Fig. 2b). Third, the mean light level for each source-detector pair was displayed as a function of the source-detector separation (Fig. 2c). The quality of source-detector coupling was evaluated through inspection of the log-linear fall-off of the light-level plots as a function of source-detector distance. Proper coupling produces narrow variance in the light levels for a given source-detector distance while poor coupling distorts the light-level to distance relationship typically by decreasing the light levels. Fourth, a histogram of the NSR for all measurement pairs was displayed (Fig. 2d). Optimal coupling of the HD-DOT array leads to values below $7.5 \%$ in the NSR histogram. A fifth real-time readout leveraged the fact that pulsatility of the arterial blood flow is a reliable signature of strong coupling in a CW optical system ${ }^{37}$. The spatial distribution of the pulse signature was also displayed to aid in optimizing cap fit at the bedside (Fig. 2d). Displaying these metrics in real time helped the user optimize cap fit within 10 minutes, thereby maximizing data acquisition time for the study.

\section{Clinical structural imaging}

All of the evaluated stroke patients $(n=13)$ underwent a head CT scan as part of their standard clinical care. The CT scans were performed during the $48-72$ hours following stroke onset using a Neurologica 
CereTom portable CT scanner $(1.25 \times 1.25 \times 10 \mathrm{~mm})$ or a Siemens Sensation Open scanner $(0.5 \times 0.5 \times$ $3 \mathrm{~mm}$ ) depending on patient stability and scanner availability. The CT scans were then evaluated by a board-certified neurologist to define a patient-specific ischemic infarct mask. Of the evaluated CT scans, masks were able to be generated for nine of thirteen patients. Of the remaining four patients without visible infarct on CT, one patient underwent a brain MRI as part of standard clinical care (Siemens Symphony TIM, TRA Diffusion, $0.9 \times 0.9 \times 6 \mathrm{~mm}$ ). This MRI was evaluated by the same neurologist as the CT scans, and an infarct mask was generated. The surface projections of these 10 masks were combined to generate an infarct incidence map (Fig. 1d).

\section{HD-DOT Data Preprocessing and Reconstruction}

Source-detector measurements that exhibited a standard deviation $<7.5 \%$ of their mean signal were considered low-noise measurements and retained for further processing. The $10 \mathrm{~Hz}$ log-ratio sourcedetector measurements were band-pass filtered to $0.009-0.08 \mathrm{~Hz}$. Superficial and systemic hemodynamic variance was removed through regression of the averaged low-noise first nearest-neighbor measurements ${ }^{38}$. First, second, and third nearest-neighbor measurement data were down-sampled from $10 \mathrm{~Hz}$ to $1 \mathrm{~Hz}$ and used for the image reconstruction. Volumetric reconstructions of absorption coefficients at both 750 and $850 \mathrm{~nm}$ were obtained using an inverted sensitivity matrix generated with an atlas representation of the adult head (Montreal Neurological Institute (MNI) 152-subject ICBM non-linear registration atlas ${ }^{39,40,41}$ ). The adult atlas-based model of sensitivity was calculated using the NIRFAST light modeling toolbox ${ }^{42}$, utilizing an FEM mesh based on the previously described head tissue segmentation and optical properties for scalp, skull, CSF, gray and white matter 36,43 . Three-dimensional maps of relative concentration changes of oxyhemoglobin $\left(\Delta \mathrm{HbO}_{2}\right)$ and deoxyhemoglobin $(\Delta \mathrm{HbR})$ were computed using the extinction coefficients of each hemoglobin species 44,45 . Finally, the data were smoothed with a $12 \mathrm{~mm}$ FWHM Gaussian kernel and transformed into MNI atlas space. Because the sensitivity profile decays exponentially as a function of depth, the effective penetration is limited in this system to approximately $2 \mathrm{~cm}$ below the head surface, corresponding to a threshold in the top $90 \%$ of the inverted sensitivity profile ${ }^{35}$.

\section{Automated Motion Detection and Censoring}

Ideal cap fits do not guarantee high quality data. Noise in the data, such as system fluctuations or patient motion, can be misinterpreted as brain fluctuations and in particular, can cause erroneous functional connectivity results, as in $\mathrm{fMRI}^{46}$. Noisy measurements from system issues were identified by having a temporal standard deviation greater than 7.5\% (Fig. 2c) and were removed before image reconstruction as detailed above. Additionally, we utilized a time-point specific motion detection algorithm, termed global variance of the temporal derivatives (GVTD) ${ }^{47}$ that is analogous to the DVARS metric of fMRI ${ }^{48,49}$ ). The GVTD metric is calculated as the root mean square across measurements of the temporal derivative in light levels. Temporal sections of data were classified as clean when they exhibited a GVTD value below an empirically defined threshold of 0.0015 for at least 60 contiguous seconds. This fast and automated cropping of motion-contaminated data was performed using the optical measurements before 
reconstruction into the voxelated space to maximize sensitivity of the metric as described by Sherafati et al., 2020. Each epoch of measurement data that remained after the automated motion detection was then individually pre-processed and reconstructed as previously published ${ }^{35}$.

\section{Functional Connectivity Analyses}

Individual participant's epochs of clean and filtered volumetric $\Delta \mathrm{HbO}_{2}$ were concatenated together. The global signal, averaged over the entire HD-DOT field of view, was regressed from every voxel ${ }^{35,38}$. Region of interest time traces, corresponding to a given seed location, such as the motor cortex, were computed by averaging the voxels within a $5 \mathrm{~mm}$ radius sphere centered at a given seed location (Fig. 3). Seedbased zero-lag fc-maps were created by calculating the Pearson correlation coefficient between the seed time trace and time trace of every other voxel within the field of view. Correlation coefficients were Fisher$Z$ transformed before further analysis. Given the high dimensionality of the data (10,241 fc-maps per subject with 10,241 voxels each), a similarity metric was calculated to reduce dimensionality. The similarity metric for a given seed location was generated by calculating the spatial Pearson correlation coefficient between the fc-map of interest (e.g. stroke patient or older normal subject) and the averaged young healthy fc-map (Fig. 4). Similarity metric maps were created by calculating similarity metrics for every seed in the field of view $(n=10,241)$.

\section{Statistical analyses}

To assess potential stroke-induced disruption in functional connectivity, we first compared individual fcmaps to a reference healthy young adult dataset. The young healthy mean reference fc-map was generated by averaging the Fisher Z-transformed fc-maps of the seven young healthy participants (Fig. 4a - lower panel). Then, for each voxel in the FOV of a particular subject (older control or stroke patients), a Pearson correlation was calculated between the respective fc-maps of the participant and the young healthy mean reference. This spatial correlation produces a similarity metric for the subject's connectivity patterns at a given seed compared to the patterns of a young healthy normal average reference. For stroke patients, datasets with infarcts in the right hemisphere were flipped across the sagittal axis in order to have all infarcts located in the left hemisphere. The Pearson correlation was then Fisher Z-transformed and assigned to the corresponding voxel location for the subject (Fig. 4b). The process was then repeated for each voxel in the FOV (Fig. 4c), and for all participants (stroke patients and older controls). To establish an average older control similarity map (upper map of Fig. 5a), the mean Fisher-Z value for a given voxel of each older control subject similarity map $(n=11)$ was calculated and then assigned to the respective voxel. This was performed for each seed location in the field of view. The same process was used to produce the mean stroke patient similarity map $(n=13)$ (Fig. 5).

To compare the mean similarity between stroke patients and older healthy controls, the mean similarity for each subject was calculated by averaging all $z$ values in each subject's similarity map to produce a single similarity summary metric per subject. These values were then compared using an independent samples t-test. We tested for differences in variance using Levene's test, which was evaluated as nonsignificant $(p=0.363)$, and so equal variances were assumed. To evaluate the effect size of the group 
comparison of fc-map similarity, Cohen's-d was calculated. The distribution of similarity values for a given stroke patient was calculated using the skewness of similarity values (Fig. 5). The relationship between the skewness of the similarity with the NIHSS of the stroke patients was calculated using Pearson correlation. For the nine patients with identifiable infarct on CT scan, a Pearson correlation was performed between the infarct volume and the NIHSS.

\section{Declarations}

\section{Author Contributions}

Author contributions: K.M.B., B.A.B., JM.L., J.P.C. and A.T.E. conceptualization; K.M.B., B.A.B., A.K.F., T.M.BY., S.L.F., R.D., G.K. and A.T.E. data curation; K.M.B., B.A.B., A.K.F., S.L.F., R.D., G.K. and A.T.E. formal analysis; K.M.B., B.A.B., A.S., B.J.P., R.D., JM.L., J.P.C. and A.T.E. methodology development; JM.L., J.P.C. and A.T.E. project administration; JM.L., J.P.C. and A.T.E. provided resources; B.A.B., A.S., S.L.F., J.P.C. and A.T.E. software development and support; JM.L., J.P.C. and A.T.E. supervision; K.M.B., B.A.B., A.S. and A.T.E. visualization; K.M.B., B.A.B., and A.T.E. drafting original document; K.M.B., B.A.B., A.S., T.M.BY., S.L.F., B.J.P., R.D., JM.L., J.P.C. and A.T.E. document review and editing; B.A.B., JM.L., J.P.C. and A.T.E. funding acquisition.

\section{Acknowledgments}

This work was supported by the National Institutes of Health (K01-MH103594 to A.T.E) (U01 EB027005, R01NS090874 to J.P.C.), International Anesthesiology Research Society (IMRA to B.A.B.), KL2 TR000450 -7 (B.J.P)

\section{References}

1. Benjamin EJ, Muntner P, Bittencourt MS. Heart disease and stroke statistics-2019 update: a report from the American Heart Association. Circulation 139, e56-e528 (2019).

2. Hacke W, et al. Thrombolysis with alteplase 3 to 4.5 hours after acute ischemic stroke. N Engl J Med 359, 1317-1329 (2008).

3. Saver JL, et al. Solitaire flow restoration device versus the Merci Retriever in patients with acute ischaemic stroke (SWIFT): a randomised, parallel-group, non-inferiority trial. Lancet 380, 1241-1249 (2012).

4. Demaerschalk BM, et al. Scientific rationale for the inclusion and exclusion criteria for intravenous alteplase in acute ischemic stroke: a statement for healthcare professionals from the American Heart Association/American Stroke Association. Stroke 47, 581-641 (2016).

5. Jauch EC, et al. Guidelines for the early management of patients with acute ischemic stroke: a guideline for healthcare professionals from the American Heart Association/American Stroke 
Association. Stroke 44, 870-947 (2013).

6. Goldstein LB, Simel DL. Is this patient having a stroke? Jama 293, 2391-2402 (2005).

7. Burns JD, Green DM, Metivier K, DeFusco C. Intensive care management of acute ischemic stroke. Emergency Medicine Clinics 30, 713-744 (2012).

8. Favilla CG, et al. Optical bedside monitoring of cerebral blood flow in acute ischemic stroke patients during head-of-bed manipulation. Stroke 45, 1269-1274 (2014).

9. Biswal B, Yetkin FZ, Haughton VM, Hyde JS. Functional connectivity in the motor cortex of resting human brain using echo-planar MRI. Magn Reson Med 34, 537-541 (1995).

10. Carter $A R$, et al. Resting interhemispheric functional magnetic resonance imaging connectivity predicts performance after stroke. Ann Neurol 67, 365-375 (2010).

11. Carter AR, et al. Upstream dysfunction of somatomotor functional connectivity after corticospinal damage in stroke. Neurorehabil Neural Repair 26, 7-19 (2012).

12. Carter AR, Shulman GL, Corbetta M. Why use a connectivity-based approach to study stroke and recovery of function? Neuroimage 62, 2271-2280 (2012).

13. Geranmayeh F, Leech R, Wise RJS. Network dysfunction predicts speech production after left hemisphere stroke. Neurology 86, 1296-1305 (2016).

14. He BJ, Snyder AZ, Vincent JL, Epstein A, Shulman GL, Corbetta M. Breakdown of functional connectivity in frontoparietal networks underlies behavioral deficits in spatial neglect. Neuron 53, 905-918 (2007).

15. Zeff BW, White BR, Dehghani H, Schlaggar BL, Culver JP. Retinotopic mapping of adult human visual cortex with high-density diffuse optical tomography. Proc Natl Acad Sci U S A 104, 12169-12174 (2007).

16. White BR, Culver JP. Quantitative evaluation of high-density diffuse optical tomography: in vivo resolution and mapping performance. Journal of Biomedical Optics 15, 026006 (2010).

17. Chalia $\mathrm{M}$, et al. Diffuse optical tomography for the detection of perinatal stroke at the cot side: a pilot study. Pediatr Res 85, 1001-1007 (2019).

18. Chalia $\mathrm{M}$, et al. Hemodynamic response to burst-suppressed and discontinuous electroencephalography activity in infants with hypoxic ischemic encephalopathy. Neurophotonics $\mathbf{3}$, 031408 (2016).

19. Bauer AQ, Kraft AW, Wright PW, Snyder AZ, Lee J-M, Culver JP. Optical imaging of disrupted functional connectivity following ischemic stroke in mice. Neuroimage 99, 388-401 (2014).

20. Orukari IE, et al. Altered hemodynamics contribute to local but not remote functional connectivity disruption due to glioma growth. Journal of Cerebral Blood Flow \& Metabolism, 0271678 × 18803948 (2018).

21. Zhang D, Raichle ME. Disease and the brain's dark energy. Nat Rev Neurol 6, 15-28 (2010).

22. White BR, et al. Resting-state functional connectivity in the human brain revealed with diffuse optical tomography. Neuroimage 47, 148-156 (2009). 
23. Eggebrecht AT, et al. Mapping distributed brain function and networks with diffuse optical tomography. Nature Photonics 8, 448-454 (2014).

24. Singh $\mathrm{H}$, et al. Mapping cortical haemodynamics during neonatal seizures using diffuse optical tomography: a case study. Neurolmage: Clinical 5, 256-265 (2014).

25. Fox MD, Greicius M. Clinical applications of resting state functional connectivity. Frontiers in systems neuroscience 4, 19 (2010).

26. Corbetta M. Functional connectivity and neurological recovery. Developmental psychobiology 54, 239-253 (2012).

27. Deco G, Corbetta M. The dynamical balance of the brain at rest. Neuroscientist 17, 107-123 (2011).

28. Carter AR, et al. Resting Interhemispheric Functional Magnetic Resonance Imaging Connectivity Predicts Performance after Stroke. Ann Neurol 67, 365-375 (2010).

29. Siegel JS, Snyder AZ, Ramsey L, Shulman GL, Corbetta M. The effects of hemodynamic lag on functional connectivity and behavior after stroke. Journal of Cerebral Blood Flow \& Metabolism 36, 2162-2176 (2016).

30. Laumann TO, et al. Functional system and areal organization of a highly sampled individual human brain. Neuron 87, 657-670 (2015).

31. Gordon EM, Laumann TO, Adeyemo B, Petersen SE. Individual variability of the system-level organization of the human brain. Cereb Cortex 27, 386-399 (2017).

32. Brott T, et al. Measurements of acute cerebral infarction: a clinical examination scale. Stroke 20, 864-870 (1989).

33. van Meer MPA, et al. Recovery of Sensorimotor Function after Experimental Stroke Correlates with Restoration of Resting-State Interhemispheric Functional Connectivity. J Neurosci 30, 3964-3972 (2010).

34. Baldassarre A, et al. Dissociated functional connectivity profiles for motor and attention deficits in acute right-hemisphere stroke. Brain 139, 2024-2038 (2016).

35. Eggebrecht AT, et al. Mapping distributed brain function and networks with diffuse optical tomography. Nat Photon 8, 448-454 (2014).

36. Eggebrecht AT, et al. A quantitative spatial comparison of high-density diffuse optical tomography and fMRI cortical mapping. Neuroimage 61, 1120-1128 (2012).

37. Wheelock MD, Culver JP, Eggebrecht AT. High-density diffuse optical tomography for imaging human brain function. Rev Sci Instrum 90, 051101 (2019).

38. Gregg NM, White BR, Zeff BW, Berger AJ, Culver JP. Brain Specificity of diffuse optical imaging: improvements from superficial signal regression and tomography. Frontiers in Neuroenergetics 2, 14 (2010).

39. Mazziotta J, et al. A probabilistic atlas and reference system for the human brain: International Consortium for Brain Mapping (ICBM). Philosophical transactions of the Royal Society of London Series B, Biological sciences 356, 1293-1322 (2001). 
40. Fonov V, Coupé P, Eskildsen S, Manjon J, Collins L. Multi-atlas labeling with population-specific template and non-local patch-based label fusion. (ed^(eds) (2012).

41. Ferradal SL, Eggebrecht AT, Hassanpour M, Snyder AZ, Culver JP. Atlas-based head modeling and spatial normalization for high-density diffuse optical tomography: in vivo validation against fMRI. Neuroimage 85, 117-126 (2014).

42. Dehghani H, Pogue BW, Poplack SP, Paulsen KD. Multiwavelength three-dimensional near-infrared tomography of the breast: initial simulation, phantom, and clinical results. Appl Opt 42, 135-145 (2003).

43. Ferradal SL, et al. Functional imaging of the developing brain at the bedside using diffuse optical tomography. Cereb Cortex 26, 1558-1568 (2015).

44. Wray NP, DeBehnke RD, Ashton CM, Dunn JK. Characteristics of the recurrently hospitalized adult. An information synthesis. Medical care 26, 1046-1056 (1988).

45. Bluestone A, Abdoulaev G, Schmitz C, Barbour R, Hielscher A. Three-dimensional optical tomography of hemodynamics in the human head. Opt Express 9, 272-286 (2001).

46. Power JD, Barnes KA, Snyder AZ, Schlaggar BL, Petersen SE. Spurious but systematic correlations in functional connectivity MRI networks arise from subject motion. Neuroimage 59, 2142-2154 (2012).

47. Sherafati A, et al. Global motion detection and censoring in high-density diffuse optical tomography. Human Brain Mapping $\mathbf{n} / \mathbf{a}$.

48. Power JD, Barnes KA, Snyder AZ, Schlaggar BL, Petersen SE. Spurious but systematic correlations in functional connectivity MRI networks arise from subject motion. Neuroimage 59, 2142-2154 (2012).

49. Smyser CD, et al. Longitudinal analysis of neural network development in preterm infants. Cereb Cortex 20, 2852-2862 (2010).

\section{Figures}

a

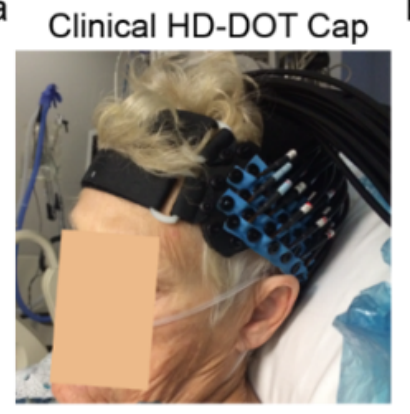

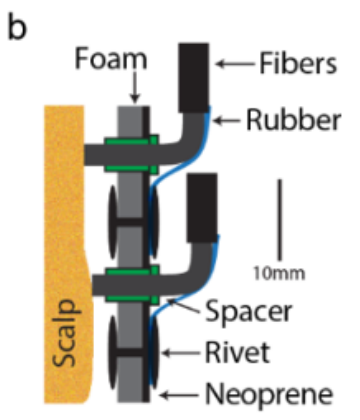

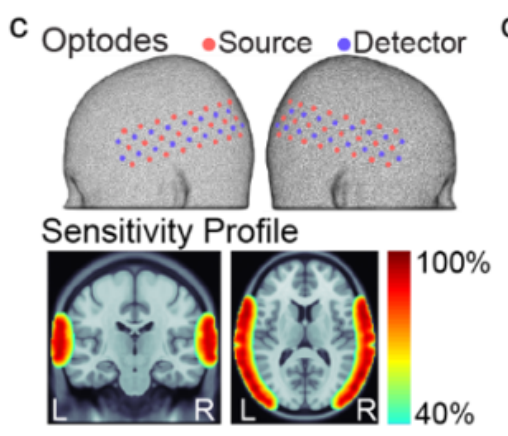

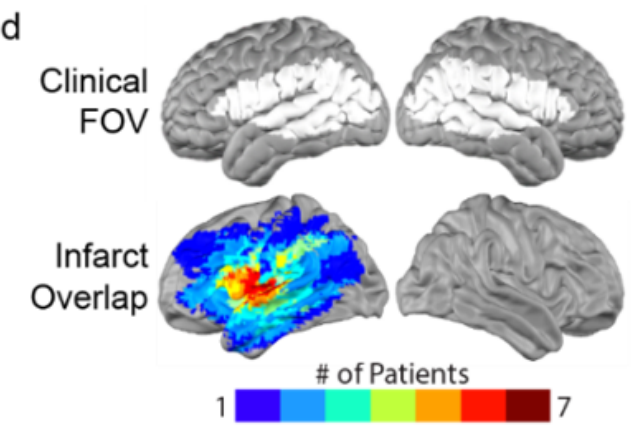

\section{Figure 1}

Clinical High-Density DOT System. a) The HD-DOT system images stroke patients at their hospital room bedside. The cap straps around the forehead with the fibers traveling upwards and crossing over the head to balance torque on the cap. The weight of the fibers is fully supported by the bed. b) The cap consists 
of two semi-rigid, rubber-like patches of neoprene located over the MCA territory. An extra layer of foam neoprene sits on the inside of the cap to add stability to the spacers, which guide the fibers through the cap. To keep the fibers pushed towards the center of the cap, rubber stripping sits tightly on the outside of the cap. Rivets hold the neoprene sheet, foam, and rubber stripping in place. c) Interlaced grids of sources and detectors sit against the side of the head just above the ears. Using source-detector separations between 1.3 and $3.9 \mathrm{~cm}$ provides uniform sensitivity. d) The field of view of the cap, shown in white, covers aspects of pre-frontal, auditory, and visual networks. The infarct incidence map for the 10 of 13 patients with identifiable infarcts on clinical scans included in the forthcoming analysis centered within the HD-DOT field of view, shown here on an inflated view of the cortical ribbon.

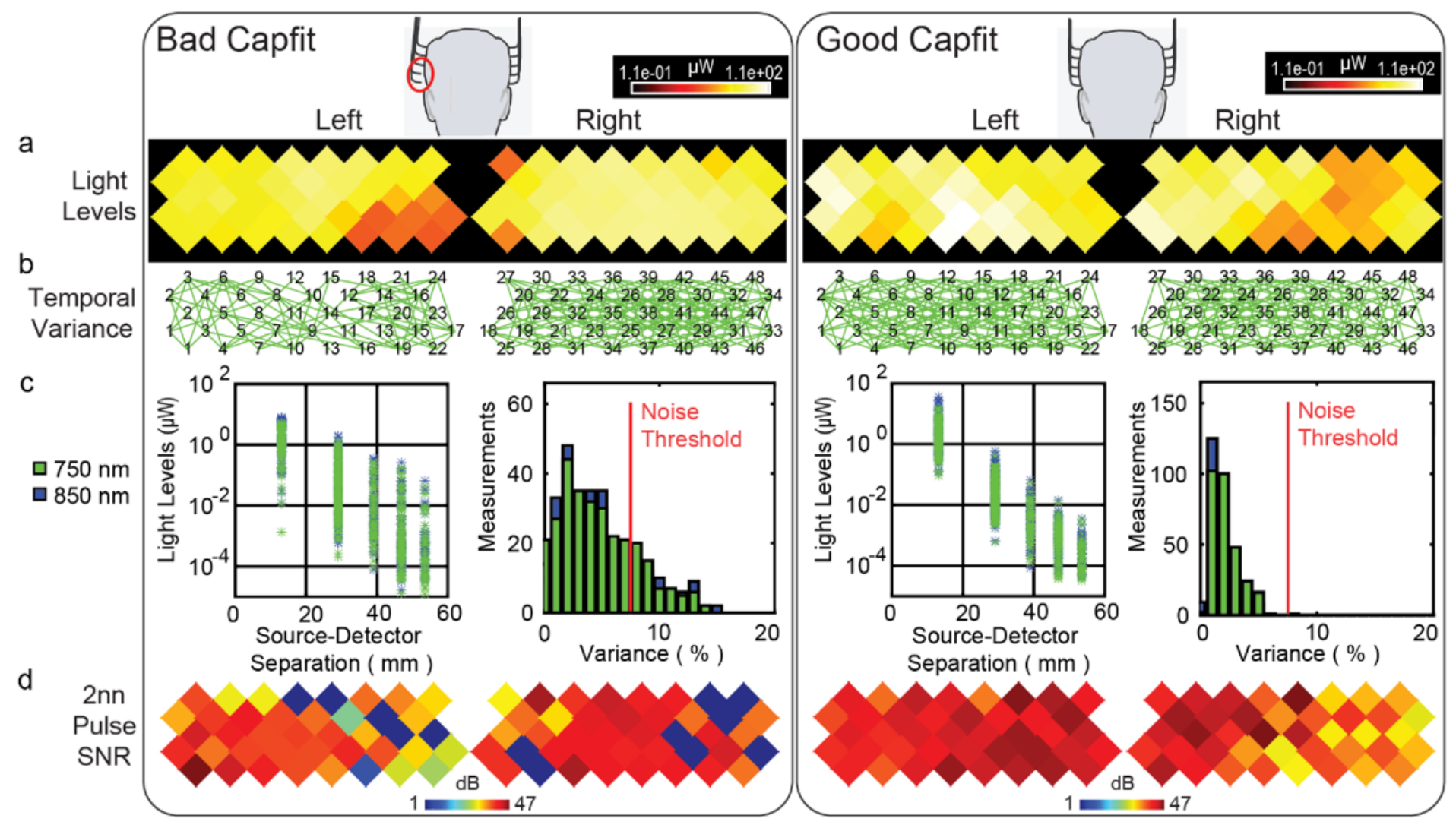

Figure 2

Real-time clinical High-Density DOT algorithms ensure high quality data. Bad cap-fits (left panel indicated by the red circle) cause a) low, non-uniform light levels since some of the fibers are successfully touching the scalp and some are not, b) loss of measurement channels on the side of poor capfit shown by missing source-detector connections due temporal variance $>7.5 \%, \mathrm{c}$ ) (left) light levels per neighbor distance that vary more than 2 orders of magnitude and have a shallow falloff with source-detector separation, (right) noisy measurements due to the fiber moving freely, and d) low SNR as measured by the pulse physiology. After modifying the cap so all fibers are touching the scalp (right panel) , a) the light levels are higher and more uniform, b) more measurements are being saved for analysis, c) (left) the light levels are within two orders of magnitude for each separation and fall off in a log-linear fashion, c) (right) 
the temporal standard deviation of all measurements is below $7.5 \%$, and d) all measurement channels have a SNR above $15 \mathrm{~dB}$ measured in the pulse frequency band.

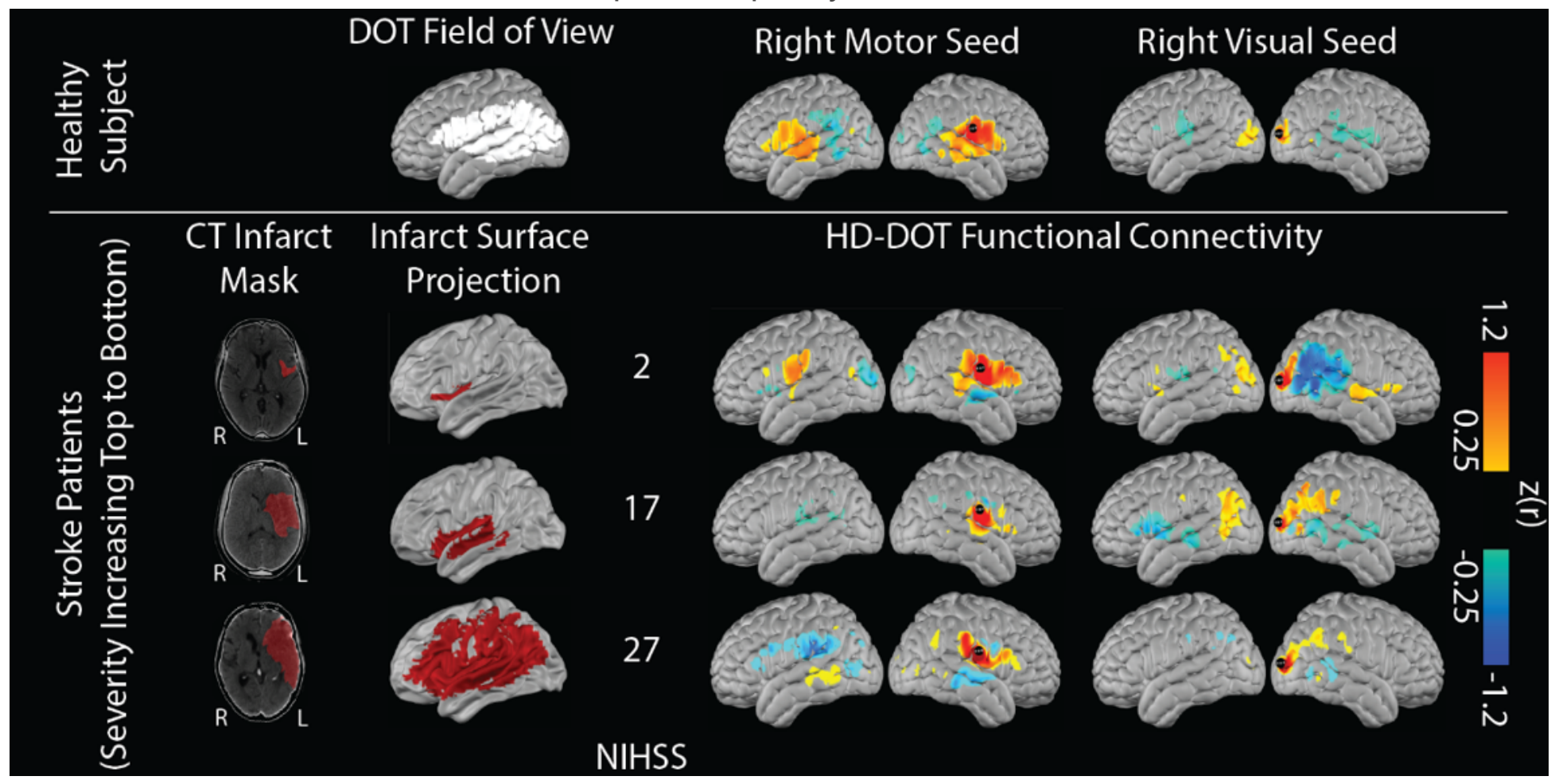

\section{Figure 3}

fcDOT in acute stroke patients. Functional connectivity (fc) maps from a healthy subject show highly correlated regions around the seed in addition to the same network on the contralateral hemisphere. Stroke patients' fc-maps for seeds placed in the right (unaffected) motor and visual cortices exhibit changes in connectivity patterns commensurate with injury. Patients with a low NIHSS have qualitatively similar bilateral patterns with high correlation values on both hemispheres whereas patients with more severe injuries show disrupted bilateral connectivity that is either close to zero or negative, indicating a loss of temporal synchrony between cortical regions of the same brain networks. 


\section{Right Motor Seed Similarity Example}

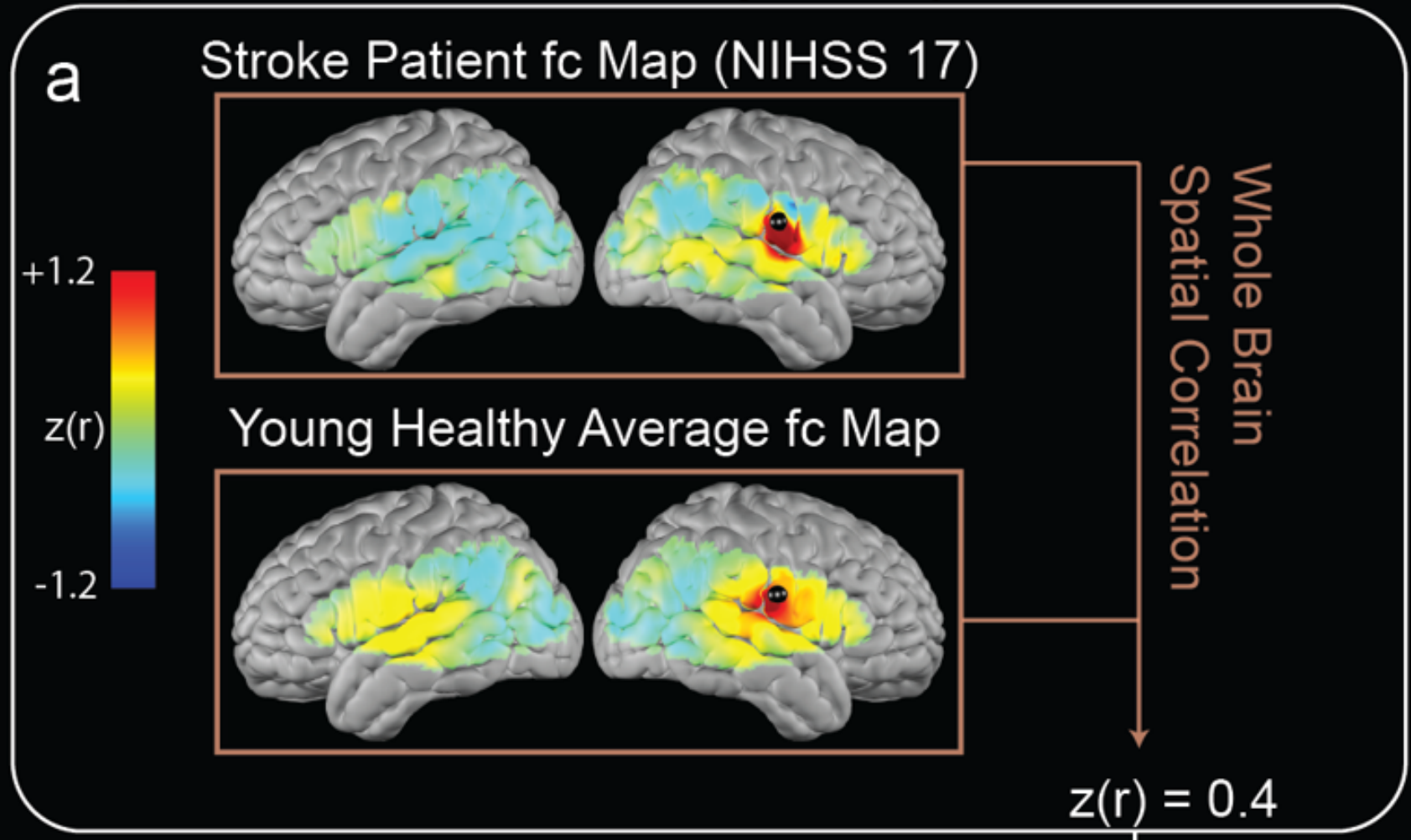

\section{Right Motor Seed Similarity Voxel}

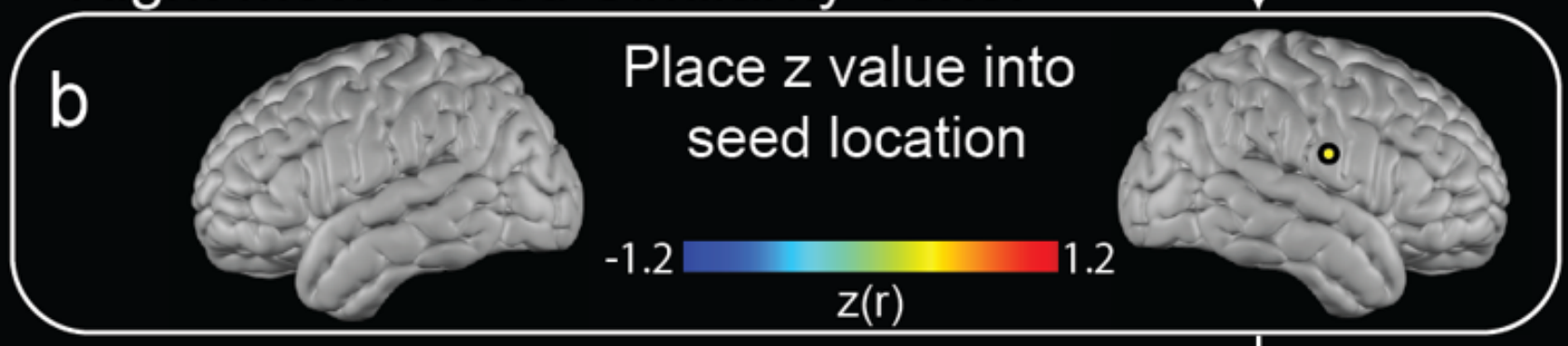

\section{Whole Brain Similarity Map}

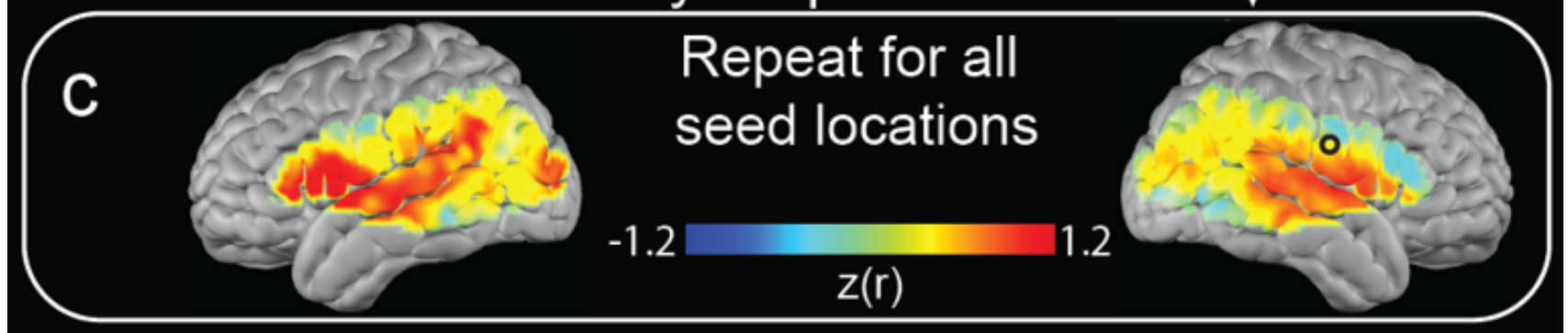

Figure 4

The similarity metric condenses all available fc-maps into one similarity map. a) The similarity value is calculated for the right motor seed. The right motor fc-maps for a stroke patient and the average young healthy fc-map are shown. The similarity metric is calculated as the spatial correlation between the two seed-based fc-maps. The resulting correlation values are the similarity for the right motor seed $(\mathrm{z}(\mathrm{r})=$ 0.4). b) To analyze all possible seed locations, that correlation value is placed into the right motor seed 
location and the process is repeated for all other seeds in the field of view $(n=10,241)$. c) This forms a whole brain similarity map.
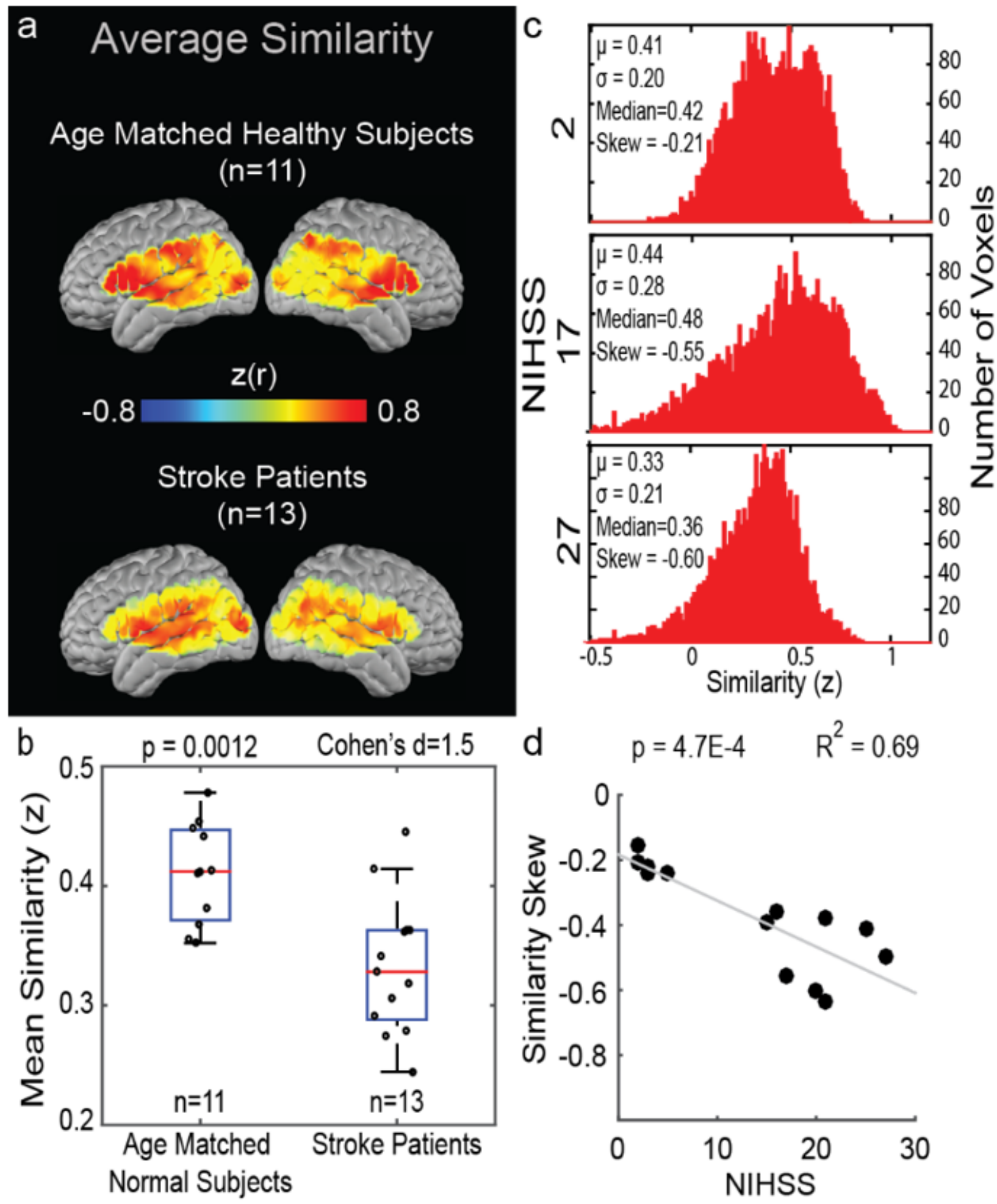

Figure 5

The similarity metric changes significantly with the presence of stroke and with stroke severity. a) Older healthy populations have more similarity to young healthy subjects than the MCA stroke patients both when visually inspecting the average similarity map across subjects, and b) when comparing the per 
subject mean similarity, where each circle corresponds to a single subject $(p=0.0012)$. $c)$ With an overall decrease in similarity values, the similarity metrics become more negatively skewed as stroke severity (measured by NIHSS) increases as shown in three representative subjects, and d) has a significant linear correlation with the NIHSS ( $n=13, p<4.7 E-4, R 2=0.69)$.

\section{Supplementary Files}

This is a list of supplementary files associated with this preprint. Click to download.

- StrokeNatureCommSupplement.docx 